\title{
Shell Lines in Be Disks
}

\author{
R. W. Hanuschik \\ Data Management and Operations Division, European Southern \\ Observatory, D-85748 Garching, Germany
}

\begin{abstract}
Shell lines have high diagnostic potential for the kinematical conditions in Be disks. We present here results from our long-term spectroscopic monitoring campaign of Fe II shell lines. We demonstrate that most of them are likely to arise in Keplerian disks being hydrostatically supported. There is furthermore one class of shell lines (dubbed NOACs) which does not fit into this simple scheme.
\end{abstract}

\section{Introduction}

The $\approx 20 \%$ fraction of Be stars observed at high inclination usually shows absorption lines in the optical spectra, with central intensity well below the stellar continuum. These are traditionally termed shell lines. They arise from absorption of stellar light in the disk and are counterparts of the troughs in P Cygnitype line profiles.

Offering a pencil-like probe of that part of the circumstellar envelope which appears projected onto the stellar surface, they have high diagnostic potential. This is especially important for determining the radial component of the velocity field in the circumstellar disk. Thus shell lines can help to decide whether Be star disks are dominated by Keplerian rotation, or by radial outflow or inflow motions.

\section{Observations and classification}

We present here results from our long-term (up to 13 years), high-resolution $\left(\Delta v=3-6 \mathrm{~km} \mathrm{~s}^{-1}\right)$ spectroscopic monitoring campaign of more than $100 \mathrm{Be}$ and shell stars. These data were mostly taken at the $1.4 \mathrm{~m}$ CAT (ESO), with contributions from the $1.5 \mathrm{~m}$ OHP, $2.2 \mathrm{~m}$ Calar Alto and $2.2 \mathrm{~m}$ Xinglong (PR China) telescopes. Part of the data has been published (Hanuschik et al. 1996).

We focus here on optical Fe II shell lines, mostly Fe II $\lambda 5317$. These are perfect tracers of disk conditions since their emission is (almost) optically thin and their thermal width at $10^{4} \mathrm{~K}$ is only $1.7 \mathrm{~km} \mathrm{~s}^{-1}$, as compared to typical values of $400 \mathrm{~km} \mathrm{~s}^{-1}$ for the rotational broadening.

We find the following classes of Fe II shell lines:

- symmetric, broad, shallow (width of order $2 v \sin i$, central depth $\geq 0.9$ );

- symmetric, broad, deep (width of order $2 v \sin i$, central depth $\leq 0.6$ );

- symmetric, narrow, shallow (width $\approx 10^{-2} v \sin i$, central depth $\geq 0.9$ );

- asymmetric and deep;

- narrow optical absorption components (NOACs). 


\section{Towards interpretation}

Broad and shallow $F e$ II lines. These show the 'standard' shell line profile (Fig. 1a). This is expected from a 'compact' $\left(R_{\mathrm{d}} \leq 5 R_{\star}\right)$ Keplerian disk absorbing stellar light, seen under inclination close to $90^{\circ}$. Their width is expected to be of order $2 v \sin i$ (Hanuschik 1995). Their depth is expected to rest above $\approx 0.8$ for disk radii in the range 5-10 $R_{*}$. In our data, none of the Fe II lines has shown the central quasi-emission peaks predicted by our simple theory for those disks. Such features, being signatures of the outer disk radius, have indeed been observed by Rivinius et al. (1999).
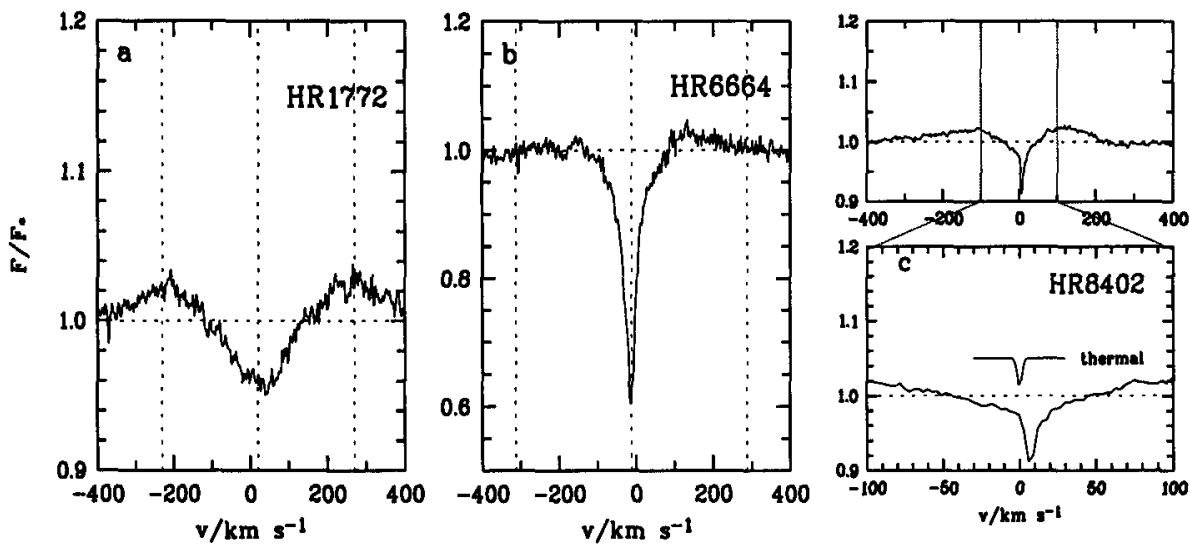

Figure 1. Examples of shell lines. (a) Broad and shallow (Fe II $\lambda 5317$ in HR 1772; measured in 930909). (b) Broad and deep (Fe II $\lambda 5169$ in HR 6664; 950411). (c) Narrow and shallow (Fe II $\lambda 5317$ in HR 8402; 941109). Vertical broken lines mark stellar rest velocity, $\pm v \sin i$

Broad and deep lines. Deep Fe II lines (Fig. 1b) require both an extended disk $\left(R_{\mathrm{d}} \approx 10 \ldots 20 R_{\star}\right)$, and broadening additional to thermal broadening. Hence these lines can be regarded as evidence for turbulent broadening in the disk.

Narrow lines: constraint on radial motions. Narrow Fe II lines (Fig. 1c) have a width of just a few thermal widths $\left(\Delta v_{\text {th }}=1.7 \mathrm{~km} \mathrm{~s}^{-1}\right)$. They are embedded in the broad, shallow component. Their shape requires a region without any radial velocity component down to that width.

They presumably arise in the outer regions of a Keplerian disk seen at inclination $70-80^{\circ}$. At that inclination range, lines-of-sight towards the stellar disk intersect the disk twice if it is of hydrostatic shape. In that case, its scale height $H(R) \propto R^{3 / 2}$, giving the disk a concave shape, as is well known from e.g. accretion disk theory. The lines-of-sight cross both the inner disk regions giving rise to the broad, shallow component, and the outer regions with radial velocity $\approx 0 \mathrm{~km} \mathrm{~s}^{-1}$. Hence the narrow shell lines can be regarded as indicators of a hydrostatic shape of the Be disk.

Furthermore, just by a variation of $R_{\mathrm{d}}$, these components can be made disappear and re-appear, which could be the origin of the famous shell events. 

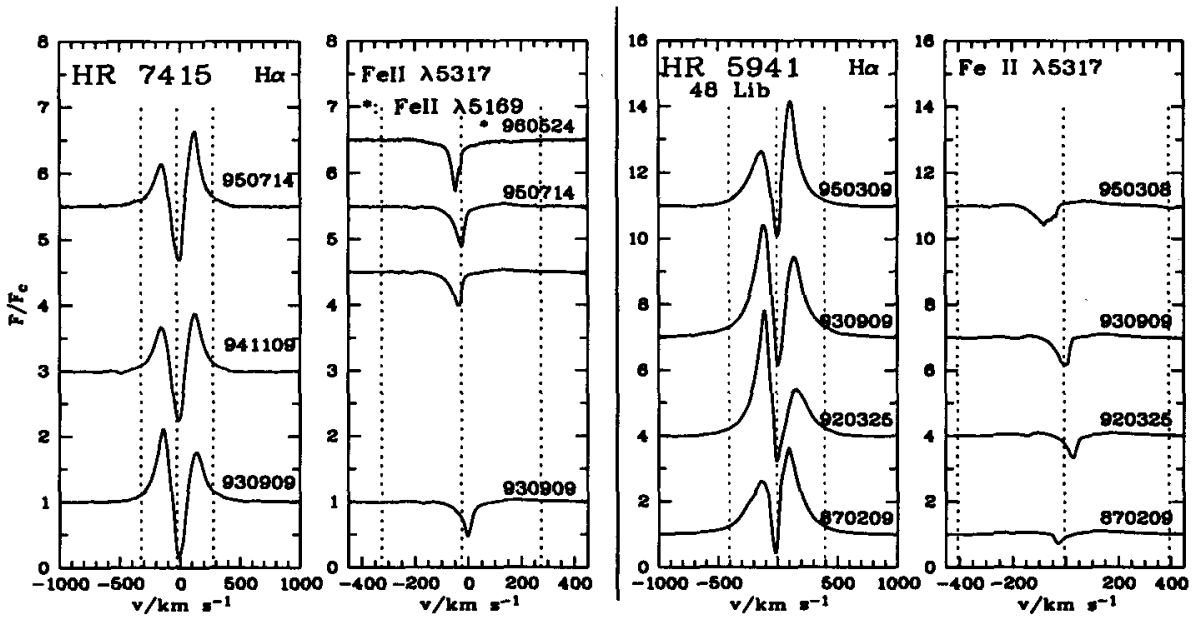

Figure 2. Asymmetric shell lines in HR 7415 (1993-1996; left) and HR $5941=48$ Lib (1987-1995; right)

Asymmetric and broad lines. This type of shell lines (see Fig. 2) shows cyclic variability in the same way as many emission lines do in Be stars (V/R variability), with a timescale of typically years. Their asymmetry follows a common pattern: that side of the shell trough which is closer to $0 \mathrm{~km} \mathrm{~s}^{-1}$ usually shows a flank steeper than the opposite one. Hence these shell lines are likely to arise in Be disks hosting a global density wave (Hanuschik et al. 1995).

NOACs: still enigmatic. In one case (48 Lib, Hanuschik \& Vrancken 1996; see Fig. 3), a further, quite unusual class of shell lines has been found: the Narrow Optical Absorption Components (NOACs). They are embedded in the broad asymmetric shell trough of Fe II lines in this star which is known to undergo cyclic $\mathrm{V} / \mathrm{R}$ variability since long. They are extremely narrow (unresolved at $R=$ $10^{5}$ ). Their shape and radial velocity varies on a timescale of hours, although the whole phenomenon persisted at least for more than a year in 1995/6. They are clearly of different origin than the stellar wind NACs since those move towards higher negative radial velocities, while the NOACs move towards lower blue radial velocities. To speculate about their origin, they could represent either higher modes in the density wave pattern, or star spots.

\section{Conclusions}

High-resolution observations of Fe II shell lines in Be stars provide strong support for Keplerian rotation being the dominant kinematical mode in Be star disks: they are usually symmetric, centered on $0 \mathrm{~km} \mathrm{~s}^{-1}$, can become very narrow down to a few thermal widths. If asymmetric, they show cyclic variability, indicating a distorted Keplerian disk hosting a density wave. The existence of an extremely narrow Fe II shell component supports a hydrostatic shape of Be disks. There is evidence for turbulent broadening in addition to thermal broadening. 

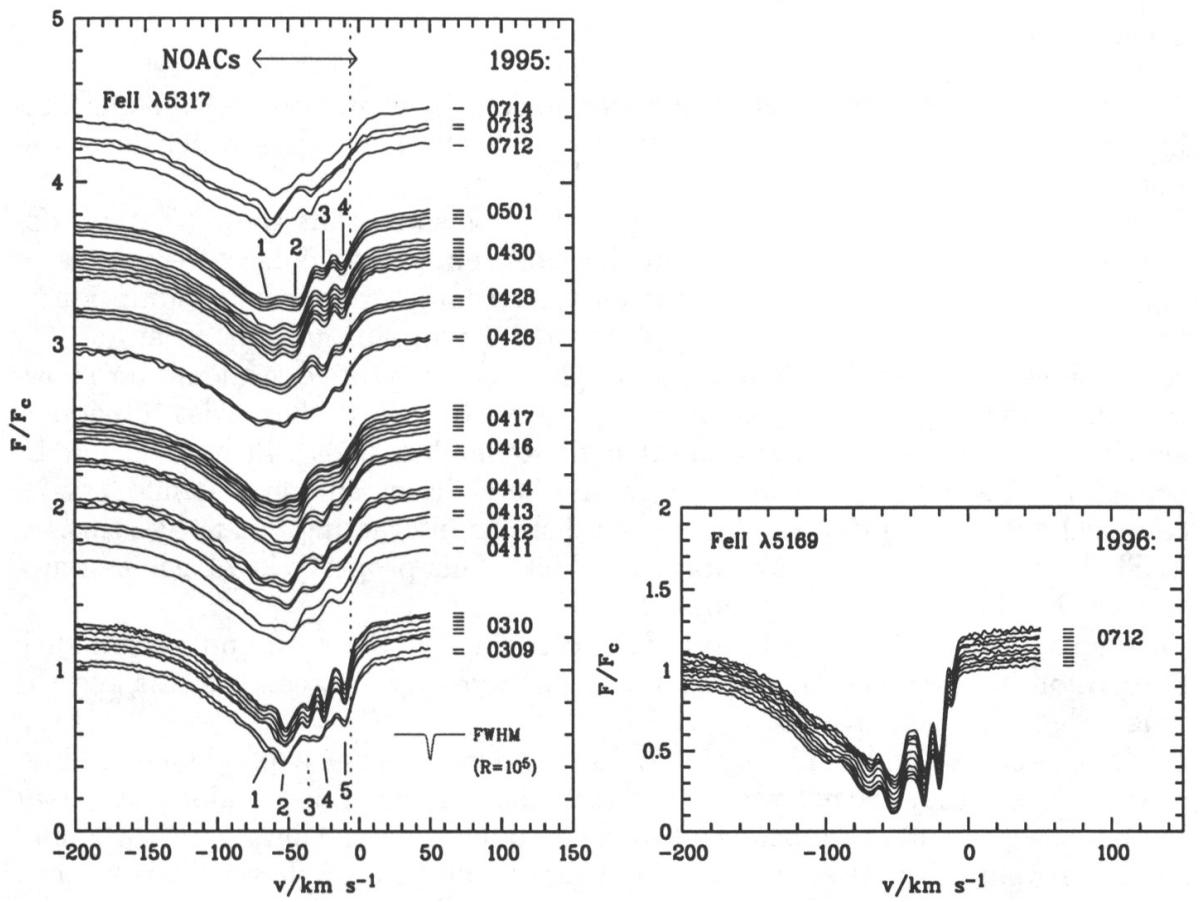

Figure 3. Fe II $\lambda 5317$ NOACs in Fe II lines of 48 Lib in 1995 (left) and 1996 (right). The FWHM given applies for the 950310 and all 960712 data only, for the other profiles $R=50000$. Numbers refer to single NOACs. The 1996 data cover 8 hours

There is no evidence for large-scale outflows or inflows in the shell volume. This argues against stellar wind models as e.g. the wind-compressed disk model (Bjorkman \& Cassinelli 1993). This does not exclude small-scale radial drift caused by viscous forces.

\section{References}

Bjorkman, J.E., Cassinelli, J.P. 1993, ApJ 409, 429

Hanuschik, R.W. 1995, A\&A 295, 423

Hanuschik, R.W., Hummel, W., Dietle, O., Sutorius, E. 1995, A\&A 300, 163

Hanuschik, R.W., Vrancken, M., 1996, A\&A 312, L17

Hanuschik, R.W., Hummel, W., Sutorius, E., Dietle, O., Thimm, G. 1996, A\&AS 116, 309

Rivinius, Th., Štefl, S., Baade, D. 1999, A\&A, in press 


\section{Discussion}

J. Zorec: a) Can you explain the appearance of spectroscopic shell signatures in those Be stars with rather permanent shell characteristics which have low $v \sin i$ values?

b) Self-absorption curves of Fe II line multiplets show that these lines are optically thick in Be stars. To explain the emission components of Fe II lines, is their optically thin character in your model actually a necessary assumption?

R. Hanuschik: a) I could not explain shell features from pole-on stars. My conclusions are derived from a large sample of objects, none of them ever showing the above mentioned phenomenon. Such objects, if they exist, obviously would require absorptive material at high stellar latitudes which could not be provided by a disk. Shell features in the here discussed sense should not be confused with absorption satellites from disks in interacting binary systems.

b) Shell lines are by definition optically thick. This property is a basic assumption of the Hanuschik (1995) models.

D. Baade: Can your explanation of the combination of broad and narrow shell absorption lines be used to constrain any possible gap between the star and the inner edge of the disk?

R. Hanuschik: I'm afraid, no. Shell lines integrate values $\exp (-\tau)$ along linesof-sight, hence they are not very sensitive to the innermost disk regions. A gap at the inner edge would result in the broad component being slightly narrower and shallowing out a bit. Best evidence for a gap would be a single very narrow shell component, without the broad shell component. This we have never observed, though.

A.M. Hubert: In the case of $\zeta$ Tau metallic lines such as Fe II are often double. They show a broad, main component and sometimes a sharp and narrow secondary component. This sharp component has a radial velocity highly variable, not close to zero (see poster by Ballereau et al.). Could you comment on this?

R. Hanuschik: The line profiles of $\zeta$ Tau look much different from most others in our atlas (Hanuschik et al. 1996). They have great similarity, though, with those of HR 2142. Both objects are interacting binaries. Their shell lines appear to me as arising from quasi-Keplerian disks (in case of $\zeta$ Tau with a one-armed distortion) plus a more complicated, variable distortion by the companion. Such profiles hence are far more difficult to interpret and require e.g. phase-binned observations (cf. HR 2142 in Hanuschik et al. 1996). Furthermore, it may be misleading to call the subordinate features in $\zeta$ Tau 'shell features'. I prefer to reserve this term for features well below the stellar continuum. The satellite features in $\zeta$ Tau could equally well be subordinate emission minima. 